\title{
The Safety of Immediate Postoperative Mitomycin C Installation: Is it a Myth? Our Experience with Consequent Peri Uretero-Vesical Junction Involvement
}

\author{
Barak Rosenzweig ${ }^{1 *}$, Dorit E Zilberman ${ }^{1}$, Tomer Erlich ${ }^{1}$, Roy Morag${ }^{1}$, Zohar Dotan ${ }^{1}$, Eddie Fridman², Jacob \\ Ramon ${ }^{1}$, Yoram Mor ${ }^{1}$ \\ ${ }^{1}$ Departments of Urology, Sackler School of Medicine, Tel-Aviv University, Israel \\ ${ }^{2}$ Department of Pathology, Sackler School of Medicine, Tel-Aviv University, Israel
}

Submission: December 29, 2016; Published: February 01, 2017

*Corresponding author: Barak Rosenzweig, Department of Urology, Sackler School of Medicine, Tel-Aviv University, Israel, Tel: 00-972-3-5302231; Email: barak22@gmail.com

\begin{abstract}
Background: Transurethral resection of tumor (TURT) followed by one immediate postoperative instillation of chemotherapeutic agent such as Mitomycin C (MMC) is considered by many as the treatment of choice for non-muscle invasive bladder cancer (NMIBC). However, when bladder perforation occurs during TURT, postoperative instillation may lead to extra-vesical leakage and consequent severe morbidity. Despite the increasingly published warnings regarding this potential dismal side effect, it has been our impression that it has not gained adequate awareness and that relevant precautious measures have not been sufficiently implemented in the common clinical practice.
\end{abstract}

Objectives: To reinforce prior observations regarding potential hazardous complications of early post-TURT instillation of MMC.

Materials \& methods: A retrospective review of surgery \& pharmacy log books for patients treated with TURT \& immediate post operative MMC was conducted in an attempt to identify and characterize postoperative cases of peri uretero-vesical junction involvement.

Results: Between the years 2009-2010, 392 cases of TURT were performed in our institute, of which 19 (4.8\%) were subsequently treated with immediate post-TURT MMC. Three of 19 (16\%) suffered major post-operative complications due to unsealed perforations and peri-ureteral reaction, necessitating prolonged renal drainage. Two patients have eventually undergone major surgeries.

Conclusion: Severe side effects necessitating prolonged hospitalization, continuous urinary drainage and sometimes even repeat extended operations, may complicate the immediate installation of MMC after TURT in cases with either recognized or unrecognized bladder perforations. Whenever the possibility of bladder perforation is suspected, avoidance of immediate post-operative instillations of MMC may be prudent.

Keywords: Superficial bladder cancer; Intravesical chemotherapy; Complications; Mitomycin C

Abbreviations: TURT: Transurethral Resection of Tumor; MMC: Mitomycin C; NMIBC: Non-Muscle Invasive Bladder Cancer; IPIC: Immediate Postoperative Instillation of Chemotherapeutic Agent; EAU: European Association of Urology; AUA: American Urological Association; TCC:

Transitional Cell Carcinoma; BCG: Bacillus Calmette Guerin; SPC: Supra Pubic Catheter; DJS: Double J Stents

\section{Introduction}

Bladder cancer is the $9^{\text {th }}$ most common cancer diagnosis worldwide, with more than 330,000 new cases diagnosed each year and more than 130,000 deaths per year. At the initial diagnosis of bladder cancer, $70 \%$ of cases are diagnosed as nonmuscle-invasive bladder cancer (NMIBC) and approximately $30 \%$ as muscle-invasive disease [1]. Transurethral resection of bladder tumor (TURT) is the initial treatment for visible lesions $[2,3]$.

The adjuvant treatment with one immediate postoperative instillation of chemotherapeutic agent (IPIC), such as Mitomycin
C (MMC) or Epirubicin, has been studied for nearly three decades [1]. The rationale behind this strategy is to destruct any residual circulating tumor cells and prevent their implantation in the bladder wall. Impressive decreases of up to $50 \%$ in tumor recurrence rates after one immediate postoperative instillation have been published [1,4]. Understandably, the current European Association of Urology (EAU) guidelines recommend one, early, postoperative intra-vesical instillation of chemotherapeutic agent followed by further instillations according to risk stratification [3]. In accordance, the contemporary American Urological Association (AUA) Guidelines for bladder cancer 
published in 2007 also advocate this strategy since «several randomized trials and recent meta-analyses have demonstrated a relative-risk reduction with a single peri-operative dose of MMC in patients with non-muscle invasive urothelial carcinoma with both low- and high-risk features» [2].

Nevertheless, an immediate postoperative instillation is not always completely harmless. Review of the guidelines discloses that both EAU and AUA expert panels are aware of reports of a «small number of serious complications related to MMC extravasation» [2] and thus recommend that «the immediate post-operative chemotherapy instillation should be omitted in any case of overt or suspected intra or extra-peritoneal perforation» [3]. However, despite the increasing awareness among the guideline panel members, it has been our impression that this warning is based upon very few reported cases and that the consequent precautious measures have not been sufficiently implemented in the common clinical practice.

\section{Materials and Methods}

We have retrospectively reviewed our surgery log book between 2009-2010, and identified all TURT's preformed. We cross matched this data with our pharmacy database and identified patients treated with immediate post-TURT MMC. Of particular interest were those cases that were treated with immediate post-TURT MMC and experienced major post- operative complications.

\section{Results}

Between the years 2009-2010, 392 patients have undergone TURT's, of which 19 (4.8\%) were postoperatively treated with immediate postoperative MMC instillation. Of these 19, 3 cases (16\%), experienced fulminant complicated postoperative courses which are described herein.

The first case was of a 63 year old female with past history of documented superficial bladder transitional cell carcinoma (TCC) and repeat TURT's followed by multiple Bacillus Calmette Guerin (BCG) and MMC courses underwent formal TURT for multiple papillary lesions of different sizes and subsequent immediate intra-vesical installation of $40 \mathrm{mg}$ MMC. She was discharged on postoperative day 2 and re-admitted 4 days later due to abdomino-pelvic pain and lower abdominal tenderness. CT cystography showed findings consistent with extra-peritoneal bladder perforation necessitating urethral catheter placement. Following catheter removal, a month later, her abdominal pain re-emerged and repeat CT cystography showed persistent extravasation emanating from the anterior bladder wall. Explorative laparotomy showed diffuse peritonitis and peri-vesical inflammation. Purulent fluid was drained and a supra pubic catheter (SPC) was inserted. A vesico-cutaneous fistula developed following SPC removal (Figure 1).
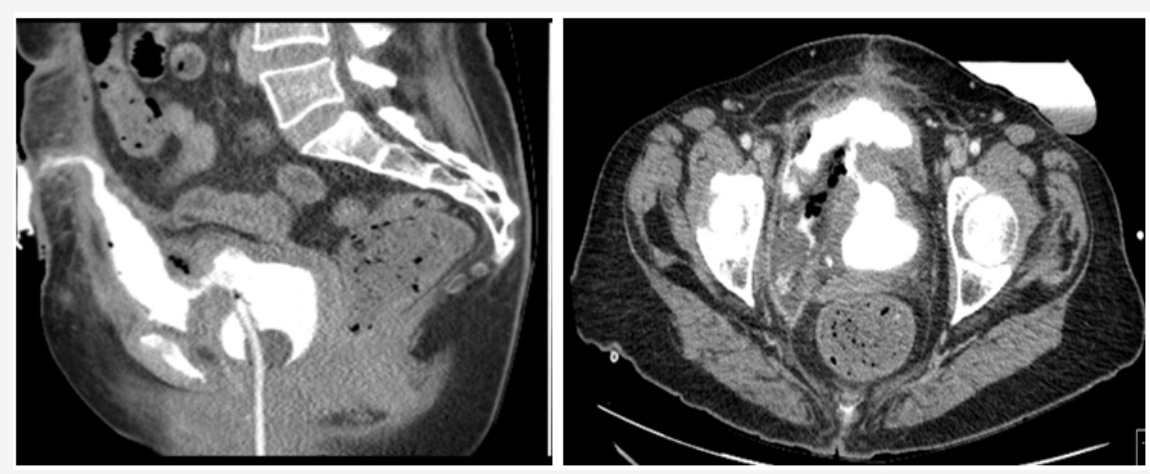

Figure 1: CT-Cystography: Sagittal (1-A) \& Transverse (1-B) sections showing remarkable leakage of contrast material from the anterior bladder wall draining into a right pelvic collection and a cutaneous fistula.
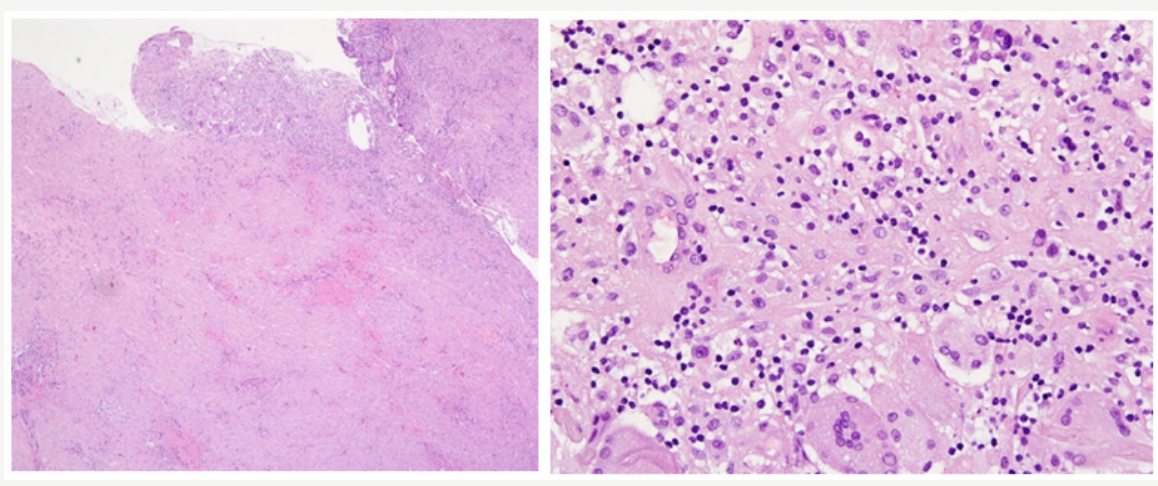

Figure 2: Acute and chronic inflammation, prominent fibrosis, and urothelial erosion (2-A); Granulation tissue formation with numerous foreign body type multinuclear histiocytes (2-B). 
Bilateral nephrostomies were inserted in order to divert the urine but the patient kept on suffering from recurrent abdominopelvic pain accompanied by persistent fistula. Antegrade nephrostogram showed distal right ureteral obstruction. Six months following her last TURT and MMC installation, a second elective explorative laparotomy was carried out due to chronic pain and persistent fistula. Again, diffuse perivesical inflammation, adhesions of small, partially obstructed bowel, as well as an ongoing posterior and anterior bladder perforation were observed. Perforated sites were widely excised, formal bladder closure was preformed and double J stents (DJS) were bilaterally inserted for 6 weeks. Postoperative course was uneventful and pathological findings showed previous biopsy changes associated with acute and chronic inflammation, with no evidence of malignancy (Figure 2).

The second case was of a 74 year old male with past history of upper urinary tract TCC treated by right laparoscopic nephroureterectomy was diagnosed as having bladder TCC and was referred for TURT. At surgery, multiple superficial, papillary lesions were completely resected and $40 \mathrm{mg}$ of MMC were subsequently administered in the recovery room for 1 hour. Four days postoperatively, the patient complained of severe pelvic and lower abdominal pain suggesting an overlooked bladder perforation, CT scan confirmed the diagnosis which was treated by bladder drainage through an indwelling urethral catheter. Hydronephrosis was not demonstrated on CT or US at that stage. Two weeks later there was no leak demonstrated on a follow-up CT scan, the urinary catheter was removed and the patient was discharged. Subsequent cystoscopy preformed 1.5 month postoperatively failed to locate the Lt. ureteral orifice though the blood creatinine levels were unchanged. Two month postoperatively a bone scan showed mild urinary retention at the Lt kidney \& ureter. A CT scan preformed 5 months later showed a thickened bladder wall on the left side with a remarkable ipsilateral hydroureteronephrosis. A nephrostomy tube was inserted and an antegrade pyelography showed left distal ureteral obstruction. In the following months the pain persisted and the patient suffered from recurrent episodes of urinary tract infections (UTI's) and hematuria, treated by antibiotics. Seven months following his first TURT the patient underwent a repeat similar procedure in order to better characterize the bladder wall thickening seen previously on CT scan. A solid mass occupying the left bladder wall was noted while the left ureteral orifice could not be identified. Biopsies showed chronic inflammation with signs of "follicular" lymphocytic cystitis and slight excess of eosinophils. Due to the overall poor health status of the patient, no further surgical interventions are planned and he is currently carrying a left nephro-ureteral stent which is periodically exchanged.

The third case recorded was of a 61 year old male patient underwent formal TURT and subsequent immediate intra-vesical installation of MMC. Histological findings were compatible with high grade Ta TCC. During the postoperative course, following urethral catheter removal, pain and irritative urinary symptoms appeared. Urethral catheter was re-inserted resulting in symptomatic improvement. Nevertheless, due to persistence of some pain and irritative complains, a CT scan was preformed 2 months following surgery. Perivesical infiltration was demonstrated. Cystography showed urinary leakage emanating from the left posterior bladder wall. Three months postoperatively an exploratory laparotomy was carried out showing perivesical abscess draining into the bladder. Partial cystectomy and abscess drainage were preformed. Histopathological examination showed extensively inflamed fibroadipous tissue. On the 4 th postoperative day a nephrostomy tube was inserted due to left lank pain and associated hydronephrosis. Three months later an antegrade nephrostogram demonstrated distal left ureteral obstruction. Due to irritative complains lasting four months following the laparotomy an urodynamic exam was undertaken showing low volume bladder. Ten months following the above discussed TURT and subsequent MMC instillation the patient underwent bladder augmentation and re-implantation of the left ureter with an uneventful postoperative course. Histopathological examination showed moderate to severe chronic and focally active inflammation of the left distal ureteral segment as well as prominent bladder fibrosis.

\section{Discussion}

MMC is an anti-neoplastic antibiotic produced from Streptomyces caespitosus. It is one of the bi/tri-functional alkylating agents causing cross-linking of DNA and inhibition of DNA synthesis. It is rapidly cleared from the serum after intravenous administration. Clearance is affected primarily by metabolism in the liver while approximately $10 \%$ is excreted unchanged in the urine [5]. However, because of its moderately high molecular weight $(329 \mathrm{kd})$ there are only few known problems with transurothelial absorption (such as myelosuppression) [2]. It is currently considered active in treatment of breast, head and neck and non-small cell lung cancers [6].

In superficial bladder cancer an immediate postoperative intra-vesical treatment with one instillation of a chemotherapeutic agent after TURT is considered to be safe and effective method to reduce recurrence rate $[7,8]$. This therapy is nowadays presented in the guidelines of the EAU and in Campbell's Urology as the state of the art policy, while in the AUA guidelines it is mentioned as a therapeutic option [1-3].

Despite the apparently safe profile of the various intravesical agents, severe complications have been reported in the past 2 decades. These were mainly attributed to intraoperative bladder perforations, although late IPIC-related perforations have been postulated as well [7,9-16]. Oddens, et al. [17] described 3 cases of serious complications after one immediate postoperative instillation of intra-vesical Epirubicin, including long lasting frequency, severe abdominal pain and small bowel obstruction leading to laparotomy (case 1) and 
even to one death. In accordance, Tyritzis, et al. [9] described 2 cases of IPIC complications with Epirubicin including late bladder perforation with subsequent laparotomy. Regarding MMC, perivesical fat necrosis was described in a series of radical cystectomies previously treated with intra-vesical chemotherapy (not necessarily immediate) [18], while Bolenz, et al. [7] have reviewed 4 case reports describing severe late complications after MMC maintenance therapy [19-22]. Likewise, clinical data regarding severe adverse events after IPIC with MMC have been sporadically described in the English literature since 2000. Overall, 10 consecutive case reports documented severe adverse events of long duration in the form of either chronic bladder wall ulceration or cystitis associated with perivesical/ perirectal or even corpora spongiosal inflammatory response and fat necrosis, 5 of which have eventually led to exploration, open fistula closure and even cystectomy [10-14,16,23-26].

Interestingly, in all 3 cases reported herein the perivesical inflammatory process involved the vesicoureteral junction or was adjacent to the region of the distal ureter, resulting in ureteric obstruction and associated ipsilateral hydronephrosis appearing 2-5 months post -TURT. Similar observations were noted by Oehlschlager, et al. [12] describing complicated, late postoperative course involving ureteral orifice obstruction by localized benign inflammatory reaction which was successfully treated by TUR of the scarred tissue and insertion of a ureteral stent.

The idea of possible catastrophic postoperative course is especially daunting in the light of the emerging trend in the literature to increasingly adopt other approaches of perioperative instillation of MMC in some other clinical therapeutic scenarios. For example, Di Stasi, et al. [27] propose to use intravesical electromotive drug administration immediately before TURT, instead of afterwards, which might similarly leak if it has not been completely removed from the bladder before surgery. Another treatment paradigm which might become popular is to prevent bladder tumors after nephroureterctomy for primary upper urinary tract TCC by a single, late, intra-vesical chemotherapy instillation given at catheter removal, when there might still be unrecognized subclinical extravasations [28].

Based upon the aforementioned reports few authors have already suggested avoiding IPIC in cases of overt or suspected bladder perforation and a clear warning about the potential hazardous complication which might result in this scenario has been added to the contemporary American and European guidelines since 2002 [2,3,29]. As this complication has been encountered in different clinical situations, not particularly following multiple previous urological interventions or extraordinary deep or extensive resections, it is hard to characterize the population at risk. Being familiar with the fact that the actual incidence of unrecognized bladder perforations following TURT might exceed 60\%, [30] we recommend to withhold IPIC whenever even the slightest suspicion of perforation passes in the surgeons' mind.

\section{Conclusion}

Severe side effects necessitating prolonged hospitalization, continuous urinary drainage and sometimes even repeat extended operations, may complicate the immediate installation of MMC after TUR in cases with either recognized or unrecognized bladder perforations. We therefore adjoin our colleagues recommending avoidance of IPIC whenever the possibility of bladder perforation is suspected.

\section{References}

1. Stephen Jones J, Steven C Campbell (2007) Non-Muscle-Invasive Bladder Cancer (Ta, T1, and CIS). Endoscopic Surgical Management. In: Wein, Kavoussi, Novick, Partin, Peters (Eds.), Campbell's Urology ( $9^{\text {th }}$ edn). Philadelphia, USA.

2. Hall MC, Chang SS, Dalbagni G, Pruthi RS, Seigne JD, et al. (2007) Guideline for the management of non muscle invasive bladder cancer (stages Ta, T1, and Tis): 2007 update. J Urol 178(6): 2314-2330.

3. Babjuk M, Oosterlinck W, Sylvester R, Kaasinen E, Böhle A, et al. (2011) European Association of Urology (EAU). EAU guidelines on nonmuscle-invasive urothelial carcinoma of the bladder, the 2011 update. Eur Urol 59(6): 997-1008.

4. Rajala P, Kaasinen E, Raitanen M, Liukkonen T, Rintala E, et al. (2002) Finn bladder Group. Perioperative single dose instillation of epirubicin or interferon-alpha after transurethral resection for the prophylaxis of primary superficial bladder cancer recurrence: a prospective randomized multicenter study-Finn Bladder III long-term results. J Urol 168(3): 981-985.

5. Mitomycin C (2004) Pub- Chem open chemistry database 2004.

6. Bradner WT (2001) Mitomycin C: A clinical update. Cancer Treat Rev 2001 27(1): 35-50.

7. Bolenz C, Cao Y, Arancibia MF, Trojan L, Alken P, et al. (2006) Intravesical mitomycin $\mathrm{C}$ for superficial transitional cell carcinoma. Expert Rev Anticancer Ther 6(8): 1273-1282.

8. Volpe A, Racioppi M, D'Agostino D, Cappa E, Filianoti A, et al. (2010) Mitomycin $\mathrm{C}$ for the treatment of bladder cancer. Minerva Urol Nefrol 62(2): 133-144.

9. Tyritzis SI, Stravodimos KG, Mihalakis A, et al. (2009) Complications associated with primary and secondary perforation of the bladder following immediate instillations of epirubicin after transurethral resection of superficial urothelial tumours. Int Urol Nephrol 41(4): 865-868.

10. Cliff AM, Romaniuk CS, Parr NJ (2000) Perivesical inflammation after early mitomycin C instillation. BJU Int 85(4): 556-557.

11. Nieuwenhuijzen JA, Bex A, Horenblas S (2003) Unusual complication after immediate postoperative intra-vesical mitomycin $\mathrm{C}$ instillation. Eur Urol 43(6): 711-712.

12. Oehlschläger S, Loessnitzer A, Froehner M, Hakenberg OW, Manseck A, et al. (2003) Distal ureteral stenosis after early adjuvant intra-vesical mitomycin C application for superficial bladder cancer. Urol Int 70(1): 74-76.

13. Racioppi M, Porreca A, Foschi N, Delicato G, Destito A, et al. (2005) Bladder perforation: a potential risk of early endovesical chemotherapy with mitomycin C. Urol Int 75(4): 373-375.

14. Shapiro O, Jones K, Wang C, et al. (2006) Risk of post-operative intravesical mitomycin $C$ instillation following transurethral bladder tumor resection. Can J Urol 13(6): 3317-3320. 
15. Koya MP, Simon MA, Soloway MS (2006) Complications of intra-vesical therapy for urothelial cancer of the bladder. J Urol 175(6): 2004-2010.

16. Lim D, Izawa JI, Middlebrook P, Chin JL (2010) Bladder perforation after immediate postoperative intra-vesical instillation of mitomycin C. Can Urol Assoc J 4(1): 1-3.

17. Oddens JR, van der Meijden AP, Sylvester R (2004) One immediate postoperative instillation of chemotherapy in low risk Ta, T1 bladder cancer patients. Is it always safe? Eur Urol 46(3): 336-338.

18. Doherty AP, Trendell-Smith N, Stirling R, Rogers H, Bellringer J, et al. (1999) Perivesical fat necrosis after adjuvant intra-vesical chemotherapy. BJU Int 83(4): 420-423.

19. Liu CC, Chou YH, Huang CH, Tsai KB (2001) Bladder wall calcification after intra-vesical chemotherapy with mitomycin $\mathrm{C}$ - a case report. Kaohsiung J Med Sci 17(5): 274-277.

20. Cuervo Pinna MA, Cuervo Pinna C, Macias Castillo S (2001) Lung sarcoidosis following instillation of mitomycin $\mathrm{C}$ in the urinary bladder. Ann Med Int 18(12): 641-643.

21. Brady JD, Assimos DG, Jordan GH (2000) Urethral slough: a rare and previously unreported complication of intra-vesical mitomycin. J Urol 164(4): 1305 .

22. Drago PC, Badalament RA, Lucas J, Drago JR (1989) Bladder wall calcification after intra-vesical mitomycin $C$ treatment of superficial bladder cancer. J Urol 142(4): 1071-1072.

23. Neulander EZ, Lismer L, Kaneti J (2000) Necrosis of the glands penis: a rare complication of intra-vesical therapy with mitomycin. C J Urol 164(4): 1306.
24. Clark T, Chang SS, Cookson MS (2002) Eosinophilic cystitis presenting as a recurrent symptomatic bladder mass following intra-vesical mitomycin C therapy. J Urol 167(4): 1795.

25. Dini Di Stefano D, Urdaneta Pignalosa G, Rodríguez Faba O, J Huguet pérez, J Palou redorta, et al. (2008) Severe cystitis and perivesical collection posterior to Mitomycin C immediate instillation in a patient with inadverted bladder perforation]. Actas Urol Esp 32(7): 759-762.

26. Fazlioglu A, Tandogdu Z, Kurtulus FO, Parlakkilic O, Cek M (2009) Perivesical inflammation and necrosis due to mitomycin $\mathrm{C}$ instillation after transurethral resection of bladder tumor: we must be vigilant! Urol Int 83(3): 362-363.

27. Di Stasi SM, Valenti M, Verri C, Liberati E, Giurioli A, et al. (2011) Electromotive instillation of mitomycin immediately before transurethral resection for patients with primary urothelial nonmuscle invasive bladder cancer: a randomized controlled trial. Lancet Oncol 12(9): 871-879.

28. O’Brien T, Ray E, Singh R, Coker B, Beard R, et al. (2011) Prevention of bladder tumours after nephroureterectomy for primary upper urinary tract urothelial carcinoma: a prospective, multicentre, randomized clinical trial of a single postoperative intra-vesical dose of mitomycin $\mathrm{C}$ (the ODMIT-C Trial). Eur Urol 60(4): 703-710.

29. Oosterlinck W, Lobel B, Jakse G, Malmström PU, Stöckle M, et al. (2002) Guidelines on bladder cancer. Eur Urol 41(2): 105-112.

30. Balbay MD, Cimentepe E, Unsal A, Bayrak, Koç A, et al. (2005) The actual incidence of bladder perforation following transurethral bladder surgery. J Urol 174(6): 2260-2262.

\begin{tabular}{l} 
Your next submission with Juniper Publishers \\
will reach you the below assets \\
- Quality Editorial service \\
- Swift Peer Review \\
- Reprints availability \\
- E-prints Service \\
- Manuscript Podcast for convenient understanding \\
- Global attainment for your research \\
- Manuscript accessibility in different formats \\
( Pdf, E-pub, Full Text, Audio) \\
- Unceasing customer service \\
Track the below URL for one-step submission \\
https://juniperpublishers.com/online-submission.php \\
\hline
\end{tabular}

